\title{
Spinal Cord Compression Due to Extramedullary Hematopoiesis in a 27-Year- Old Man with Beta Thalassemia Intermedia: A Case Report
}

\author{
Saadat $\mathrm{P}^{1}$, Alijanpour $\mathrm{Sh}^{2}$, Sotouneh $\mathrm{N}^{3}$ and Ahangar $\mathrm{AA}^{* 4}$ \\ ${ }^{1}$ Department of Neurology, Rouhani Hospital, Babol University of Medical Sciences, Babol, Iran \\ ${ }^{2}$ Emergency Medical Service center, Babol University of Medical Sciences, Babol, Iran \\ ${ }^{3}$ Department of Internal Medicine, Rouhani Hospital, Babol University of Medical Sciences, Babol, Iran \\ ${ }^{4}$ Department of Neurology, Mobility Impairment Research Center, Babol University of Medical Sciences, Babol, Iran
}

*Corresponding author: Ahangar AA, Associated Professor, Department of Neurology, Mobility Impairment Research Center, Babol University of Medical Sciences, Babol, Iran, Tel: 09111111202, E-mail: ahmadiahangaralijan@yahoo.com

Citation: Saadat P, Alijanpour Sh, Sotouneh N, Ahangar AA (2016) Spinal Cord Compression Due to Extramedullary Hematopoiesis in a 27-Year-Old Man with Beta Thalassemia Intermedia: A Case Report. J Case Rep Stud 4(3): 306. doi: 10.15744/2348-9820.4.306

Received Date: March 15, 2016 Accepted Date: June 18, 2016 Published Date: June 21, 2016

\begin{abstract}
Background: Extramedullary hematopoiesis (EMH) occurs in approximately 15\% of cases of thalassemia. Paraspinal mass of extramedullary hematopoiesis are a rare compensatory process in intermediate and severe thalassemia.

Case presentation: We present a case of a paravertebral Extramedullary hematopoiesis with a spinal cord compression in a 27-year-old man with beta thalassemia intermedia who complaint of paresis in lower extremities and progression up to umbilical level.

Conclusion: Paravertebral Extramedullary hematopoiesis although rare, should always be considered in the differential diagnosis of any upper motor neuron disease condition in patients with thalassemia and therefore appropriate studies should perform to investigate the probable paravertebral ectopic marrow before any surgical intervention.

Keywords: Spinal cord; Thalassemia; Hematopoiesis
\end{abstract}

\section{Introduction}

Extramedullary hematopoiesis (EMH) occurs commonly in patients with severe thalassemia who receive inadequate treatment. The diagnosis of thalassemia intermedia is purely clinical and is usually made after a period of observation to determine that the patient is able to maintain a satisfactory hemoglobin ( $\mathrm{Hb}$ ) level for a long time without the need for blood transfusions. The incidence of EMH in patients with thalassemia intermedia may reach up to $20 \%$, compared to polytransfused thalassemia major patients where the incidence remains $<1 \%$ [1]. Besides the usual regions of hematopoiesis, blood cells can be formed in unusual sites like the liver, spleen and lymph nodes to meet the demands of hematopoiesis [2]. There are a few reports where EMH has involved some rare places such as the perirenal and paravertebral regions, paranasal sinuses, clivus, meninges, spinal epidural spaces, prostate, adrenals, pleura, breast, thymus, kidney, sweat gland, broad ligament and retroperitoneal space [2]. This unusual phenomenon, especially when it involves the spinal cord can lead to neurological deficits [3]. A paraspinal location for the hematopoietic tissue occurs in $11-15 \%$ of cases with EMH [1].

\section{Case presentation}

A 27-year-old Iranian man, known case of beta thalassemia intermedia, presented with the complaint of paresis in lower extremities during the last three months, with recent progression to sever paraparesis and hypoesthesia in both lower extremities up to umbilical level in abdomen and bladder and bowel incontinence.

He had a positive family history of major thalassemia in his siblings.

He had no blood transfusions, had common bile duct stone, and had past history of motor accident leading to open fracture in left leg and skull fracture in his past medical history. He consumed only folic acid.

On physical examination he was pale, prominent skull deformity and had splenomegaly. Neurological examination showed normal mental status examination, and normal cranial nerve exam. In sensory examination, his light touch and pinprick. Joint position sense was impaired in lower extremities with sensory level up to umbilical zone. Romberg test was impaired. He had spastic paraparesis 
and (3-4/5) muscle power in proximal and distal muscles of both legs. His deep tendon reflexes showed increase in lower extremities but no clonus was detected. Babinski's sign was present in both sides. Above finding were compatible with diagnosis of upper motor neuron diseases (myelopathy) in lower thoracic spinal cord segment.

On admission ,his laboratory investigation showed hemoglobin $10.6 \mathrm{~g} / \mathrm{dL}$, white blood cell count $6.3 \times 10^{9} / \mathrm{L}$, platelet count $81 \times$ $10^{9} / \mathrm{L}$, mean corpuscular volume $78 \mathrm{fl}$.

Magnetic resonance imaging (MRI) of his thoracolumbar spinal cord revealed multiple paravertebral masses with rib expansion, intraspinal extension and soft tissue masses, suggestive of Extramedullary hematopoiesis or neurofibromatosis (Figure 1 and 2).

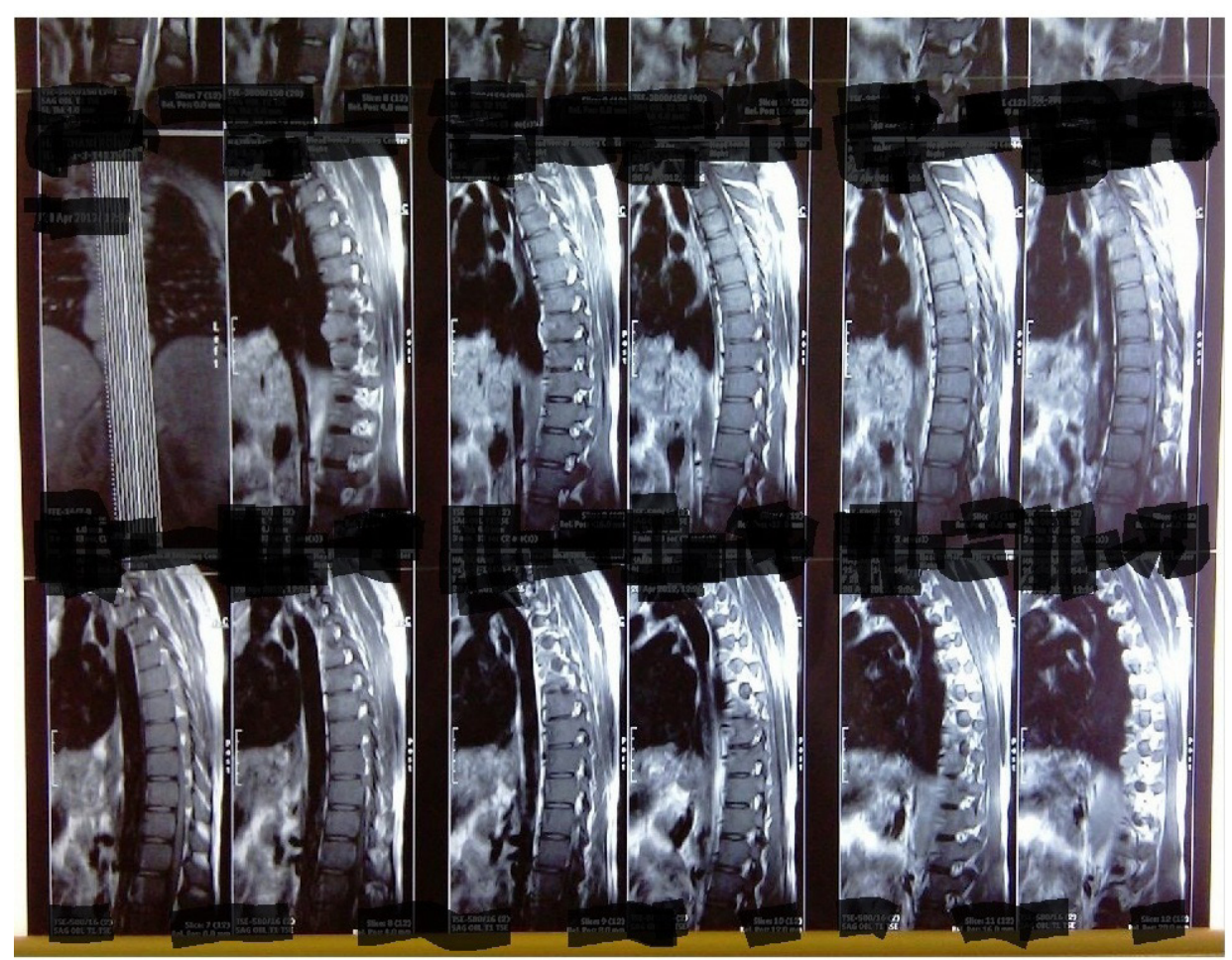

Figure 1: Saggital T1w and T2w images show a large epidural mass occupying the posterior part of the spinal canal, extending from $\mathrm{T} 5$ to $\mathrm{T} 11$ level. The mass is displacing the spinal cord anteriorly and compressing it

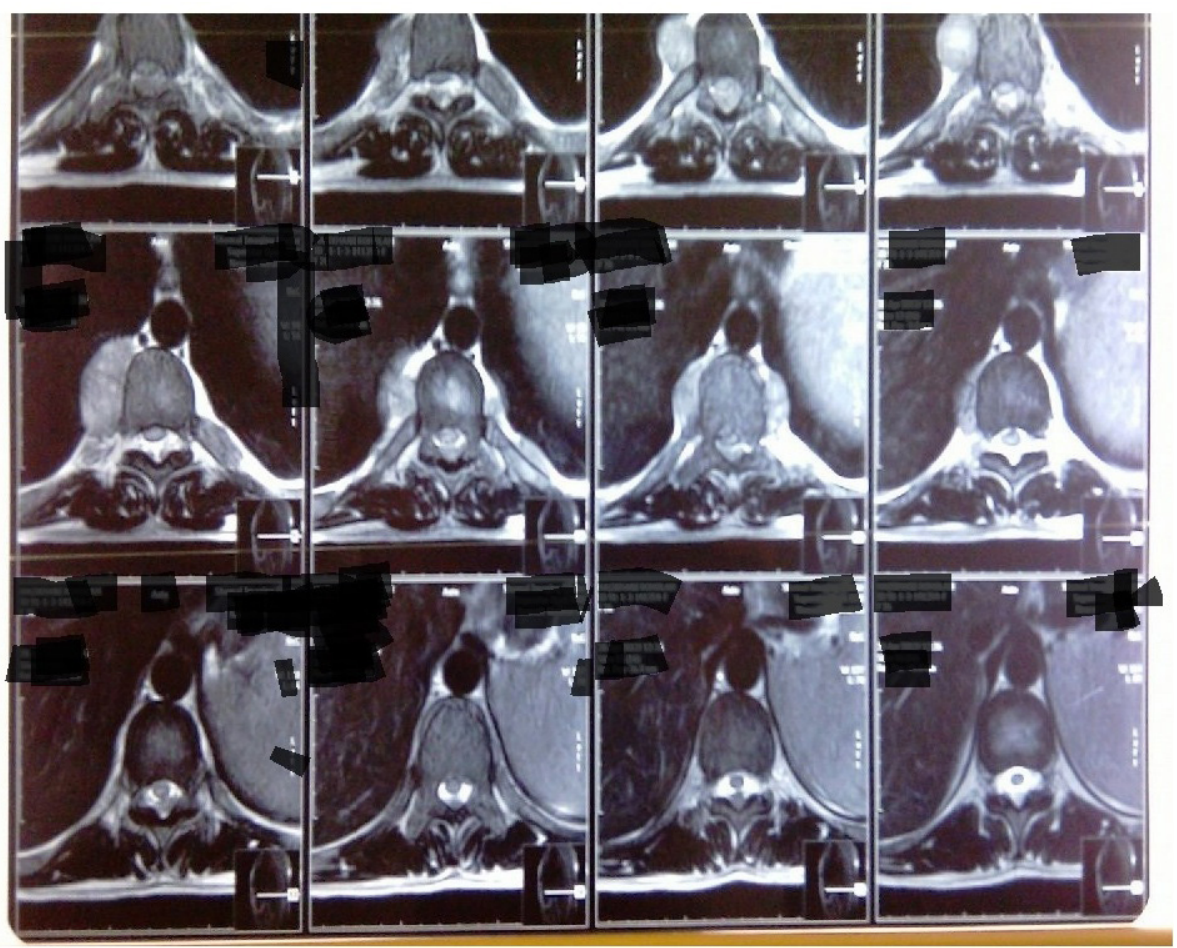

Figure 2: Axial T2w images show that the cord is flattened and displaced anteriorly by the epidural mass. In addition, the large bilateral paravertebral mass and expanded posterior ends of ribs are seen 
After doing MRI, core needle biopsy was recommended by radiologist. CT guided paravertebral mass biopsy was done and pathologic examination of specimen was compatible with hematopoietic tissue.

Macroscopically, the specimen measured $0.3 \mathrm{~cm}$ in length and was creamy and rubbery. Microscopically, the specimen was fibro connective tissue accompanied by foci of trilineages hematopoietic cells.

After the confirming of diagnosis, patient was treated by hydroxyurea $500 \mathrm{mg}$ two times a day. He showed slow recovery from the signs and symptoms of paresis and relieved his incontinency after 3 months follow up.

\section{Discussion}

Extramedullary hematopoiesis (EMH) is a compensatory phenomenon that occurs when erythrocyte production is diminished or destruction is accelerated. EMH can occur in many disorders including thalassemia, polycythaemia rubra vera, myelofibrosis, haemolytic anemia and other hemoglobinopathies. Spinal cord compression due to EMH has been reported as an uncommon complication in intermedia or major beta-thalassemia patients occurring mostly in children or young adults. EMH is usually microscopic and commonly involves the liver, spleen and lymph nodes. Thoracic manifestation is less common and consists of paravertebral soft tissue masses. The masses represent extrusion of the marrow through the thinned cortex of the posterior ribs. It usually has a predilection for the lower thoracic spine where the limited mobility and narrow spinal canal predisposes itself to spinal cord compression [4].

Most cases present with paraparesis, sensory impairment and occasionally sphincter disturbances. Complete paraplegia has been reported very rarely in thalassemia and occurs more frequently in polycythemia rubra vera and sickle cell anemia [5].The diagnostic procedure of choice is MRI which characteristically shows an isointense mass with a high signal intensity rim on T1_weighted images and a hyperintense mass on T2_weighted images. The diagnosis is based on strong clinical suspicion in the presence of diffuse bone marrow hyperplasia along with symmetric para spinal and epidural masses. Although we had performed needle biopsy to establish the definite diagnosis in this case, A core needle biopsy (CNB) is used to withdraw small cylinders (or cores) of tissue from the abnormal area. A CNB is most often done in the health care provider's office with local anesthesia. The needle is put in 3 to 6 times to get the samples, or cores. But it's more likely to give a clear result because more tissue is taken to be checked. The provider doing the CNB usually places the needle in the abnormal area using ultrasound or x-rays to guide the needle into the right place. If the area is easily felt, the biopsy needle may be guided into the tissue [1].

In some studies the non-invasive procedures such as ${ }^{18} \mathrm{~F}-\mathrm{FLT}$ PET/CT and ${ }^{99 \mathrm{~m} T \mathrm{~T}-S C}$ SPECT/CT Scintigraphy is recommended to establish the definite diagnosis [6].

Treatment options for cord compression are surgery, radiation therapy, blood transfusion, hudroxyurea or various combinations therapy. Due to the extreme rarity of this condition direct comparison between various treatments modalities are not possible. Whereas most cases presenting with paraparesis have been treated with either surgery or radiotherapy, we hereby report a case of thalassemia intermedia with paraparesis treated successfully with only drug therapy. A few authors have reported good results with the use of hydroxyurea along with hypertransfusion [7].

In report of 2cases of extra medullary hematopoiesis in the American Journal of the Medical Sciences [8], the authors have recommended to include extramedullary hematopoiesis among the differential diagnosis of tumor-like masses in patients with hematologic diseases. In this report the cases were treated without surgery. Ileri and colleague treated a 9-year-old patient suffering from thalassemia intermedia and presenting spinal cord compression with radiation therapy followed by hydroxyurea [9]. Because the risk of spinal cord injuries (myelopathy) compression due to thoracolumbar spinal that it was urgent issue, in consultation with the oncology specialist decided to treat with chemotherapy. Hudroxyurea (HU) is an inhibitor of ribonucleotide reductase and a well-tolerated cytostatic agent. Although its mechanism of action on EH has not been completely clarified yet, the clinical benefits of $\mathrm{HU}$ are well documented in patients with thalassemia and $\mathrm{EH}$. HU is a small molecule and has the potential to penetrate the central nervous system [10]. The drug, in addition to its cytostatic effects, has a favourable effect on fetal haemoglobin production. Gamberini et al. treated a 24 year old patient of thalassemia intermedia with paraplegia due to EMH, with hydroxyurea $1000 \mathrm{mg} /$ day for 5 months change to; 500mg/day up to 25 months [7]. Since our patient showed mild spinal cord compression, hydroxyurea was considered. Our patient showed rapid and near complete recovery with hydroxyurea alone despite having long standing paraparesis.

\section{Conclusion}

This case report showed that although rare, a paravertebral EMH should always be considered in the differential diagnosis of any upper motor neuron disease condition in patients with thalassemia and therefore appropriate studies performed to investigate the probable paravertebral ectopic marrow. We conclude that drug therapy may be the optimal therapeutic approach in such cases with mild paraparesis. Physicians should be taken into account the diagnosis of a congenital anemia when evaluating neurological symptoms. Once diagnosed, there is not standard care in these cases and therapeutical alternatives such us radiotherapy, surgery, hyper-transfusion or hydroxyurea must be chosen in a case-by case- manner. 


\section{Acknowledgment}

We would like to thank the staff of Neurology Department of Rouhani Hospital of Babol Medical Sciences University.

\section{Authors' Contributions}

Saadat $\mathrm{P}$ participated in the organization, writing, and final editing of this paper and approved the final version. Alijanpour S participated in the writing and final editing and approved the final version. Sotouneh $\mathrm{N}$ participated in the writing of this paper and approved the final version. Ahangar AA participated in the writing and editing of this paper and approved the final version.

\section{References}

1) Haidar R, Mhaidi H, Taher AT (2010) Paraspinal extramedullary hematopoiesis in patients with thalassemia intermedia. Eur Spine J 19: 871-8.

2) Taher AT, Musallam KM, Karimi M, El-Beshlawy A, Belhoul K, et al. (2010) Overview on practices in thalassemia intermedia management aiming for lowering complication rates across a region of endemicity: the OPTIMAL CARE study. Blood 115: 1886-92.

3) Ittipunkul N, Martin T, Siriwanasan R, Olanratanachai K, Rootman J (2007) Extra-medullary hematopoiesis causing bilateral optic atrophy in beta thalassemia/ $\mathrm{Hb}$ E disease. Med Assoc Thai 90: 809-12.

4) Tan TC, Tsao J, Cheung FC (2002) Extramedullary hematopoiesis in thalassemia intermedia presenting as paraplegia. J Clin Neurosci 9: 721-5.

5) Salehi SA, Koski T, Ondra SL (2004) Spinal cord compression in beta-thalassemia: case report and review of the literature. Spinal Cord 42: 117-23.

6) Zade A, Purandare N, Rangarajan V, Shah S, Agrawal A (2012) Noninvasive approaches to diagnose intrathoracic extramedullary hematopoiesis: 18F-FLT PET/ CT and 99mTc-SC SPECT/CT scintigraphy. Clin Nucl Med 37: 788-9.

7) Gamberini MR, Fortini M, De Sanctis V (2004) Paraplegia due to spinal cord compression by extramedullary erythropoietic tissue in a thalassaemia intermedia patient with gynecomastia secondary to cirrhosis: successful treatment with hydroxyurea. Pediatr Endocrinol Rev 2: 316-8.

8) Castelli R, Graziadei G, Karimi M, Cappellini MD (2004) Intrathoracic masses due to extramedullary hematopoiesis. Am J Med Sci 328: 299-303.

9) Ileri T, Azik F, Ertem M, Uysal Z, Gozdasoglu S (2009) Extramedullary hematopoiesis with spinal cord compression in a child with thalassemia intermedia. J Pediatr Hematol Oncol 31: 681-3.

10) Eskazan AE, Ar MC, Baslar Z (2012) Intracranial extramedullary hematopoiesis in patients with thalassemia: a case report and review of the literature. Transfusion 52: 1715-20.

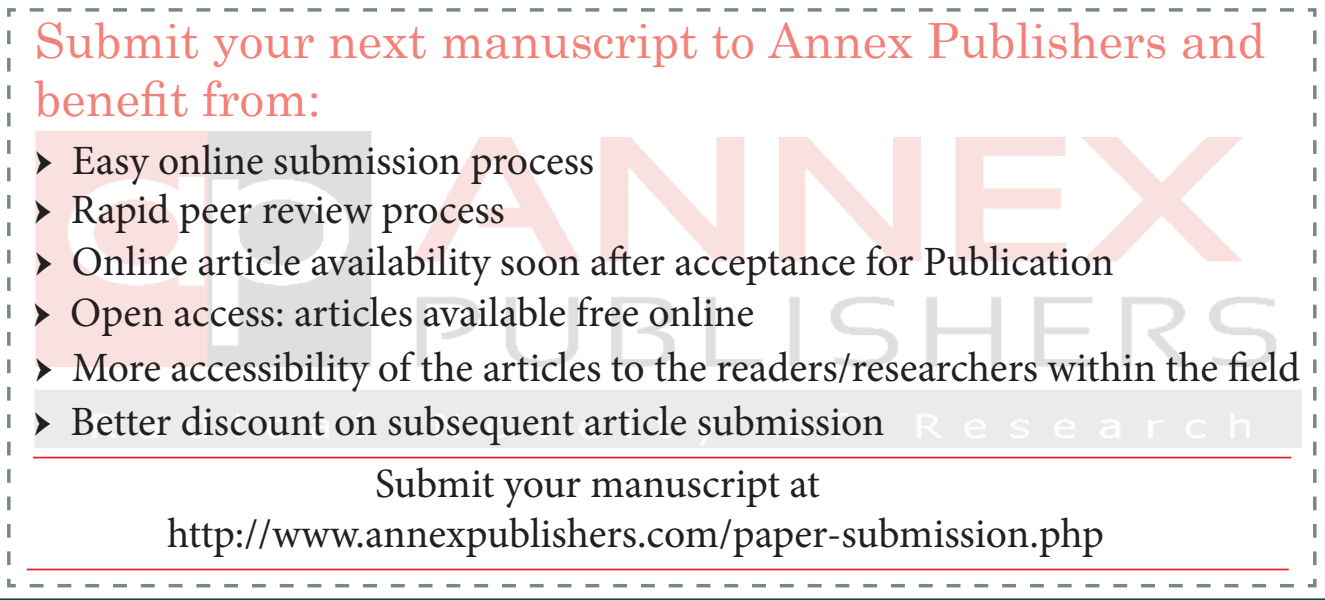

